\title{
PRÉFACE
}

\section{JOURNÉES ANGUILLES DU GIS GRISAM St-Pée-sur-Nivelle - Septembre 2003}

\begin{abstract}
Ce numéro spécial anguille du BFPP est né de la volonté de publier les travaux présentés lors des dernières journées anguilles du Gis Grisam (Groupement d'intérêt pour le suivi des amphihalins migrateurs). Les GIS ou Groupes d'Intérêt Scientifique sont des structures généralement nationales. Le Gis Grisam est né au début des années 90 de la volonté de quatre organismes publics ${ }^{1}$ de recherche ou de gestion de fédérer et d'organiser les recherches sur les amphihalins à un niveau national. Le Grisam a ainsi pour vocation de promouvoir la collaboration et la concertation entre organismes, laboratoires et/ou équipes et de formuler des avis scientifiques homogènes sur l'état des stocks des amphihalins. II est organisé en 6 groupes thématiques et le groupe Anguille est l'un de ces groupes. Son histoire est particulière au sein du Gis Grisam dans la mesure où il lui pré-existait sous l'appellation Groupe National Anguille. II fonctionne sur la base de réunions thématiques (par exemple un atelier stades pigmentaires s'est tenu sous l'égide du Grisam au Cemagref de Bordeaux au printemps 2003) et de réunions bisannuelles. Les dernières Journées Anguilles du Grisam se sont tenues à I'INRA de St-Pée-surNivelle (organisateurs : A. Bardonnet et E. Feunteun, co-secrétaires du groupe en 2003) 2 . Elles ont attiré une soixantaine de participants (gestionnaires + scientifiques) venus de toute la France. II y a eu 31 présentations (dont 6 posters), associées à des temps de discussions. Lors de la dernière après-midi, une réunion a permis de faire le bilan des données disponibles (ou d'identifier leur manque dans certains cas) et d'organiser la réflexion sur les priorités en matière de recherche autour des thèmes retenus par le CIEM (Conseil International pour l'Exploration de la Mer). Le groupe a ainsi élaboré les bases d'un document de travail pour les scientifiques qui allaient représenter la communauté française à la réunion CECPI (Commission Européenne Consultative pour les Pêches dans les eaux Intérieures)/CIEM « anguille » d'octobre 2003 (Cédric Briand, Marie-Noëlle de Casamajor, Thomas Changeux, Gérard Castelnaud). L'élaboration de ces documents de travail représente une tâche fastidieuse dans la mesure où les données sont dispersées et pas toujours facilement accessibles. Cette réflexion préalable insistait en particulier sur la nécessité de prendre en compte tous les facteurs d'origine anthropique impliqués dans le déclin de l'abondance et de l'aire de distribution de l'anguille. En particulier la réduction drastique de la surface des habitats essentiels (zones humides de la plaine d'inondation et des littoraux, ruisseaux des zones de sources), la réduction de la libre circulation des poissons réduisant l'accessibilité aux zones amont ou créant d'importantes mortalités à la dévalaison et la dégradation de la qualité de l'eau doivent être prise en considération au même titre que toutes les formes de pêches. Les réunions du Gis Grisam représentent ainsi des temps d'échange particulièrement importants pour construire des propositions et des réflexions communes avant la tenue de ces réunions.
\end{abstract}

La situation écologique de l'anguille est dramatique et les dernières tendances ne nous rassurent pas sur son avenir. En 1998, le Comité consultatif du CIEM sur la gestion des pêches a fourni des avis officiels à l'Union européenne, et en est arrivé à la conclusion que le stock d'anguilles est désormais au-delà des limites de sécurité biologique et que les pêcheries actuelles ne sont pas viables. En 2002, il était recommandé de réduire les causes de mortalité au niveau le plus bas possible tant que des mesures de gestion concertées n'étaient pas mises en place. Celles-ci sont encore aujourd'hui en cours d'élaboration,

1 Le CSP, I'Ifremer, le Cemagref et I'INRA.

2 Les prochaines journées du groupe anguille du Grisam se tiendront à Perpignan du 24 au 27 avril 2006. 
et la menace d'une fermeture partielle de la pêche pourrait accélérer le processus. Le Gis Grisam devrait occuper une place centrale dans la réflexion dans la mesure où, officiellement sollicité par le MEDD (Ministère de l'Écologie et du Développement Durable) et le MAP (Ministère de l'Agriculture et de la Pêche), il a accepté une mission d'expertise collective scientifique et technique sur ce thème. Elle sera réalisée par un large panel d'experts du « groupe anguille » du Grisam, dont l'animation est confiée à Cédric Briand (co-secrétaire du groupe depuis 2004). II devra en outre s'appuyer sur les travaux du programme INTERREG 3b INDICANG coordonné par IFREMER (Patrick Prouzet) et dont les objectifs sont de mettre en œuvre les indicateurs de l'état et de l'évolution de l'abondance et la qualité de la population d'anguilles et de l'état de l'environnement (habitats et qualité de l'eau, usages) exploité par l'espèce.

A côté de cette mission d'expertise, le Grisam a aussi orienté jusqu'en 2003 la distribution de certains crédits du MEDD (suite à des appels d'offre annuels) vers des études sur les amphihalins que le comité scientifique du Grisam avait pour mission d'évaluer. Ces appels d'offre ont malheureusement cessé à partir de 2003, ce qui a réduit le champ d'action du Gis à l'organisation des rencontres, ainsi qu'à des missions d'expertise scientifique. Les Journées Anguilles de St-Pée ont donc eu lieu à une période un peu charnière de ce point de vue, mais elles n'en ont pas pour autant été moins riches en diversité. A côté des 2 demi-journées dédiées à des discussions concernant la situation européenne et nationale de l'anguille en matière de recherche et de gestion, une journée et demi a été consacrée à des exposés scientifiques. La plus grosse session a concerné l'analyse de l'état du stock (14 présentations). Elle était organisée en 3 soussessions dédiées aux 3 phases biologiques : civelles, anguille jaune et anguille argentée. La diversité des approches a ensuite généré deux courtes sessions, l'une dédiée aux approches éthologiques des mécanismes de recrutement (3 présentations), l'autre au parasitisme (3 présentations). La dernière session intitulée «Gestion du stock, mises en œuvre et évaluation ", a permis de découvrir les différentes approches mises en œuvre sur le territoire français (Loire, Vilaine, golfe du Morbihan) pour évaluer et gérer le stock, ainsi que des approches basées sur la modélisation, ou sur la prise en compte du facteur social, approche qu'il convient de souligner étant donné son caractère novateur. Toutes ces communications scientifiques n'ont bien sûr pas été soumises pour publication au présent $\mathrm{n}^{\circ}$ spécial anguille. Cependant, les sept articles qui composent ce numéro représentent relativement bien si ce n'est en quantité, du moins en diversité, les communications présentées aux journées anguilles.

Dans leur article «Efficacité de la migration des civelles entrant dans le bassin de la Gironde en utilisant le transport tidal sélectif », Laurent Beaulaton et Gérard Castelnaud nous expliquent comment par la mise en œuvre de différentes méthodes de traitement de données des captures par pêche, ils parviennent à évaluer la vitesse de progression des civelles en estuaire. Les travaux sur "Estimation de stock de civelles en estuaire par marquage-recaptures à l'aide de colorants vitaux » de Cédric Briand, Denis Fatin, Éric Feunteun et Guy Fontenelle sur l'estuaire de la Vilaine et la passe d'Arzal mettent en évidence l'existence de 2 groupes de civelles ayant des comportements locomoteurs différents, et donc une aptitude à la colonisation particulièrement différente dans cet estuaire fermé. Dans leur article «Étude expérimentale des comportements de civelles : Influence de la densité et de la disponibilité en abris », Agnès Bardonnet, Christian Rigaud et Jacques Labonne exposent leurs travaux sur l'évolution du comportement social de la civelle et sur les conséquences possibles de ce comportement en terme de croissance et de déplacement. Dans un autre article intitulé « Effet de la réouverture d'un axe migratoire pour l'anguille (Anguilla anguilla, L.) à l'échelle d'un bassin versant », Cédric Briand, Denis Fatin, Guy Fontenelle et Éric Feunteun analysent les conséquences de la mise en place de passes à anguille sur les barrages pour la population d'anguille du bassin versant de la Vilaine. A côté des activités anthropiques défavorables à la survie de l'anguille, l'espèce a aussi à faire face à des attaques parasitaires qui peuvent comme dans le cas 
d'Anguillicola crassus, se révéler être une cause du déclin des populations d'anguille européenne à ne pas négliger. Dans leur article sur les "Étude biogéographique du parasite de l'anguille Anguillicola crassus à l'aide de marqueurs RADP ", llyesse Rahhou, Serge Morand, Raymonde Lecomte-Finiger et Pierre Sasal concluent à des arrivées multiples du parasite sur plusieurs foyers européens. Un second article sur le parasitisme est proposé par Géraldine Fazio, Raymonde Lecomte-Finiger, Jérôme Bartrina, Hélène Moné et Pierre Sasal qui évaluent la relation entre la faune parasitaire des anguilles et leur développement morphologique bilatéral comme indice de stress, dans "Communauté de macroparasites et asymétrie chez l'anguille jaune Anguilla anguilla dans la lagune de Salses-Leucate, Sud de la France ». Enfin, dans leur article "Un outil d'aide à la gestion de l'anguille dans le bassin Loire : Le tableau de bord anguille du bassin Loire " Aurore Baisez et Pascal Laffaille nous expliquent sur quelles bases, comment et pourquoi cet outil d'aide à la gestion a été mis en place.

Ce $\mathrm{N}^{\circ}$ spécial anguille devrait donc permettre au lecteur d'apprécier la diversité et la richesse des approches menées par la recherche fondamentale et appliquée pour mieux comprendre la biologie et mieux gérer les stocks d'anguille Européenne.

Agnès Bardonnet, Cédric Briand et Éric Feunteun 
